\title{
La gestión documental enfocada en la conservación preventiva de documentos
}

\section{Document Management focused on Preventive Conservation of Documents}

\author{
Luis Ernesto Paz Enrique * \\ Dirección de Recursos Humanos. Universidad Central "Marta Abreu" de Las Villas, Cuba.
}

Eduardo Alejandro Hernández Alfonso ** Departamento de Extensión Universitaria, Universidad Central "Marta Abreu" de Las Villas, Cuba

\author{
Recibido: 07 de abril de 2016 Aceptado: 07 de setiembre 2016 \\ Corregido: 06 de marzo 2017 Publicado: 12 de junio 2017
}

\section{Resumen}

La gestión documental enfocada en la conservación de documentos minimiza los perjuicios que se pueden generar en las instituciones por la pérdida o extravío. En este estudio se muestran los aspectos relativos a la gestión documental desde la conservación preventiva de documentos. Para la obtención de resultados se expone un caso concreto que se desarrolla en el ámbito administrativo, en el cual se almacenan grandes volúmenes de documentos. Se plantean como objetivos: 1) diagnosticar el estado de la documentación que se almacena en el área de formación y desarrollo de la Universidad Central Marta Abreu de Las Villas, Cuba y 2) diseñar un plan de medidas preventivas para la documentación diagnosticada. Para la obtención de resultados se emplean métodos en los niveles teóricos y empíricos. Se diagnostican los fondos de la institución, detectándose que los factores ambientales, el hacinamiento y los factores físico mecánicos son los que más influyen en la conservación de las colecciones de documentos. A partir de los resultados obtenidos se evidencia la necesidad de priorizar estrategias para el mejoramiento de las condiciones de conservación documental en la institución, especialmente en documentos que pudieran adquirir valor patrimonial.

Palabras Clave: Gestión documental, Conservación preventiva de documentos, Archivo de gestión, Almacenamiento de documentos, Universidad Central Marta Abreu de Las Villas.

\begin{abstract}
The document management focused on document preservation minimizes the damages that can be generated to the institutions by the missing or loss. The study shows the aspects related to document management from the preventive conservation of documents. To obtain results, a concrete case is presented that is developed in the administrative area where large volumes of documents are stored. The objectives of the study are: 1) to diagnose the state of the documentation stored in the area of training and development, Universidad Central Marta Abreu de Las Villas, Cuba and 2) design a plan of preventive measures for documentation diagnosed. To obtain results are used methods in the theoretical and empirical levels. The funds of the institution are diagnosed, detecting environmental factors, overcrowding and mechanical physical
\end{abstract}

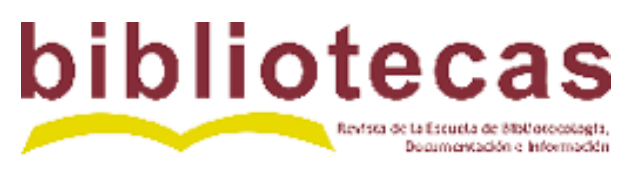


Bibliotecas. Vol 35, № 1, enero-junio, 2017, pp.1-20. EISSN: 1659-3286

URL: http://www.revistas.una.ac.cr/index.php/bibliotecas/index

factors that influence the conservation of collections of documents. From the results in evident the need to prioritize strategies for improving the conditions of conservation documentary in the institution, especially documents that may acquire heritage value.

Keywords: Document management, Preventive conservation of documents, Management archive, Document storage, Universidad Central Marta Abreu de Las Villas.

\section{Introducción}

La conservación puede definirse como el otorgamiento de un nivel apropiado de seguridad, control ambiental y almacenamiento. Es la actividad asociada al mantenimiento de materiales de bibliotecas, archivos y museos para su uso en la forma física original o en algún otro formato (microfilm, microficha, vídeo, cinta de audio, discos, entre otros). La conservación documental permite la perdurabilidad de las fuentes. La rigurosidad en la conservación permite al documento evitar la restauración, siendo esta la última etapa para salvar el contenido.

Los principios clásicos de la conservación establecen que se deben conservar los documentos en su formato original como documentos con valor legal o probatorio, libros extraños y valiosos, manuscritos originales, documentos con valor estético, documentos cuyo formato facilite la investigación (mapas, planos, plegables, documentos de gran tamaño en general) (León, 2006).

La conservación tiene un valor muy significativo para la perduración de los bienes culturales de cada región. Junto con la restauración surge para definir la disciplina en todo el ámbito patrimonial. Según Paz y Hernández (2015) "es el conjunto de operaciones que tienen por objeto prolongar la vida de un material" (p. 2). Se utiliza para designar centros oficiales con actividad científica, docente e investigativa.

Los documentos contribuyen a satisfacer las necesidades de información previamente identificadas por usuarios o grupos de ellos. La necesidad de preservar información relevante ha conllevado el desarrollo de métodos y técnicas con la finalidad de conservar la documentación. Esta conservación de documentos comprende las estrategias y técnicas 
Bibliotecas. Vol 35, № 1, enero-junio, 2017, pp.1-20. EISSN: 1659-3286

URL: http://www.revistas.una.ac.cr/index.php/bibliotecas/index

específicas, relativas a la protección de los materiales frente al deterioro, los daños y el abandono (Rose, 2014; Paz, Alfonso, Izquierdo y Brito, 2016).

La conservación documental ha sido abordada por varios especialistas, fundamentalmente por profesionales de la información, biología y de ciencias químicas. Es necesario la aplicación de estudios interdisciplinares que favorezcan la obtención de resultados lo más concreto posibles.

\section{Antecedentes y problema de investigación}

A nivel internacional, los procesos de conservación documental están regidos por la UNESCO bajo el documento Memoria del mundo. Directrices para la salvaguardia del patrimonio documental. Otros documentos que rigen este proceso son Labor del Comité del Patrimonio Mundial sobre una estrategia global, Políticas del Escudo Azul, y Recomendación de la UNESCO sobre la salvaguardia y conservación de las imágenes en movimiento. Se analizan estudios que por su objeto se relacionan con la investigación. Por su relevancia y actualidad se destacan los autores: Rivera (2009), Delgado (2011), González, Térmens y Ribera (2012), Giménez (2013), Cabezas (2014), Muñoz (2014), Paz, Hernández y Cuellar (2015).

Los documentos orgánicos envejecen naturalmente y comienzan a deteriorarse desde su génesis. Los procesos de deterioro de los documentos orgánicos pueden acelerarse en un medio ambiente adverso y sobre todo por el descuido en su manipulación. Deben evitarse las condiciones desfavorables de almacenamiento y ubicación de los documentos localizados en bibliotecas, debido a que están compuestos principalmente por materias orgánicas.

La Universidad Central Marta Abreu de Las Villas (UCLV) atesora documentos que datan de su fundación en 1952. La documentación reviste gran importancia para la historia de la universidad, así como para la historia nacional. La institución fue declarada Monumento Nacional de la República de Cuba, debido en parte a la documentación histórica y patrimonial que atesora. Se han reportado, debido a malas prácticas con la documentación, 
Bibliotecas. Vol 35, № 1, enero-junio, 2017, pp.1-20. EISSN: 1659-3286

URL: http://www.revistas.una.ac.cr/index.php/bibliotecas/index

extravíos y pérdidas totales de la documentación del centro y la documentación patrimonial en la actualidad se encuentra diseminada por varias áreas de la universidad ante la ausencia de locales adecuados para su almacenamiento. Los expedientes de Formación y Desarrollo (FD), en dependencia con las características de la persona que estén en los documentos, pueden convertirse en patrimonio bibliográfico de la universidad.

En la actualidad el centro educacional se encuentra realizando gestiones para el funcionamiento, a nivel institucional, de un archivo central e histórico; al cual tributarán todas las instancias. Ante tal situación, cada área universitaria se encuentra realizando estudios para diagnosticar e identificar documentación con diverso valor y los factores de deterioro presentes en sus colecciones documentales.

Como parte de este proceso, la Dirección de Recursos Humanos (DRH) realiza una serie de investigaciones para diagnosticar y proponer medidas preventivas a la documentación que almacenan. El área de FD resguarda, en su archivo de gestión, toda la documentación relacionada con los expedientes de los trabajadores docentes del centro, incluyendo los expedientes en sí mismos. Dicha área carece de un diagnóstico de conservación que facilite la creación de políticas de conservación documental; y no posee un plan de medidas preventivas para la conservación de los documentos. Se plantean como objetivos del estudio diagnosticar el estado de la documentación que se almacena en el área de FD y diseñar un plan de medidas preventivas para la documentación diagnosticada.

\section{Metodología}

La investigación es de tipo aplicada. Se centra en proponer acciones, a fin de solucionar la problematiza analizada. El enfoque del estudio es mixto debido a que se auxilia de la estadística descriptiva combinada con técnicas de tipo cualitativo. Las técnicas empleadas que facilitaron la recopilación de los datos son la revisión de documentos, entrevista, observación participante, guías de observación y la triangulación de información para validar los resultados obtenidos. Se emplean métodos en el nivel teórico y empírico para la recolección de los datos, entre ellos: analítico-sintético, sistémico-estructural, históricológico, inductivo-deductivo, la observación, el análisis documental clásico y la encuesta.

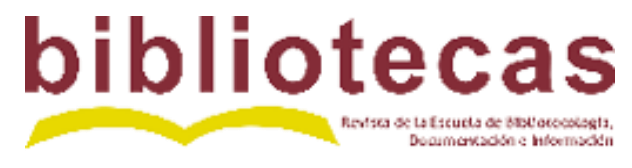


Bibliotecas. Vol 35, № 1, enero-junio, 2017, pp.1-20. EISSN: 1659-3286

URL: http://www.revistas.una.ac.cr/index.php/bibliotecas/index

Para el diagnóstico de conservación de los documentos que atesora la institución se empleó el Modelo de la Biblioteca Nacional de Venezuela, pues esta institución funciona como el Centro Regional de Conservación de la Organización de las Naciones Unidas para la Educación la Ciencia y la Cultura (UNESCO) de América Latina y el Caribe. Se emplea esta metodología dada su funcionalidad para identificar los elementos que influyen en la conservación de los documentos orgánicos y las necesidades de conservación preventiva en bibliotecas y archivos. La metodología empleada consta de seis variables, las mismas pueden observarse en la Tabla 1.

Tabla 1

Modelo para el diagnóstico de sedes y colecciones de la Biblioteca Nacional de Venezuela

\begin{tabular}{|c|l|}
\hline Variable & \multicolumn{1}{|c|}{ Descripción } \\
\hline Sede & Se refiere al espacio físico de la entidad \\
\hline Depósito & Características físicas del depósito \\
\hline Mobiliario & Condiciones físicas del mobiliario \\
\hline $\begin{array}{c}\text { Condiciones ambientales } \\
\text { del depósito }\end{array}$ & $\begin{array}{l}\text { Condiciones de iluminación, ventilación, temperatura y } \\
\text { humedad relativa }\end{array}$ \\
\hline $\begin{array}{c}\text { Condiciones generales de } \\
\text { la colección }\end{array}$ & $\begin{array}{l}\text { Se refiere a la identificación de los factores de deterioro } \\
\text { identificados }\end{array}$ \\
\hline $\begin{array}{c}\text { Medidas de prevención } \\
\text { existentes }\end{array}$ & $\begin{array}{l}\text { Medidas contra catástrofes, insectos y roedores, } \\
\text { políticas para la consulta de la documentación, } \\
\text { seguridad y vigilancia }\end{array}$ \\
\hline
\end{tabular}

Fuente: León (2006).

Se emplearon para la identificación de las afectaciones en los documentos, la leyenda de términos y abreviaturas elaborada en los Programas Diagnos y Fotodiagnos del Instituto de Historia de Cuba. Diagnos y Fotodiagnos son programas automatizados que se pueden utilizar para diagnosticar el estado de conservación de las colecciones de documentos con soporte en papel y fotográfico. Es el resultado de varios investigadores del Laboratorio de Restauración del Instituto de Historia de Cuba, además de otros profesionales de la Oficina Cubana de Propiedad Industrial. Los términos y abreviaturas de dichos programas pueden observarse en la Figura 1.

\section{bibliotecas}


Bibliotecas. Vol 35, № 1, enero-junio, 2017, pp.1-20. EISSN: 1659-3286 URL: http://www.revistas.una.ac.cr/index.php/bibliotecas/index

Figura 1

Abreviaturas utilizadas para el diagnóstico de conservación de documentos orgánicos

\begin{tabular}{|l|l|}
\hline \multicolumn{2}{|l|}{ Parámetros (leyenda) } \\
\hline PM & Pulpa Mecánica \\
\hline PQ & Pulpa Química \\
\hline PT & Pulpa de Trapo \\
\hline Est. & Estucado \\
\hline O & Otros \\
\hline I & Impreso \\
\hline G & Grafito \\
\hline MTA & Metaloácido \\
\hline Sint. & Sintético \\
\hline TCh & Tinta China \\
\hline M & Mecanográfico \\
\hline P & Pictórico \\
\hline O & Otros \\
\hline Reb & Reblandecido \\
\hline F & Faltante \\
\hline
\end{tabular}

\begin{tabular}{|l|l||l|l|}
\hline \multicolumn{2}{|l||}{ Parámetros (leyenda) Cont. } & \multicolumn{2}{l|}{ Parámetros (leyenda) Cont. } \\
\hline Frag. & Fragmentado & A & Amarillamiento \\
\hline R & Rotura & Emb. & Emborronado \\
\hline D & Doblez & Emp. & Empalidecido \\
\hline Perf. & Perforación & Ileg & Ilegible \\
\hline CA & Cinta Adhesiva & C & Corrosión \\
\hline PP & Papel pegado & O & Otros \\
\hline AH & Adherencia Hojas & HL & Hongo Local \\
\hline MOL & Mancha Oxido (local) & HD & Hongo Difundido \\
\hline MOD & Mancha Oxido Difundido & Pig & Pigmentación \\
\hline MA & Manchas de Agua & Desp & Desprendimiento \\
\hline Fox & Foxing & PI & Presencia de Insectos \\
\hline OM & Otras Manchas & PR & Presencia de roedores \\
\hline S & Suciedad & Exc. & Excrementos \\
\hline Acid. & Acidez & Abr & Abrasión \\
\hline & & Rest & Restaurado \\
\cline { 2 - 4 } & &
\end{tabular}

Fuente: Programas Diagnos y Fotodiagnos (Gómez, Dorta y Montes de Oca, 2008).

La población del estudio está constituida por el total de expedientes de FD que se almacenan en la DRH de la UCLV, registrándose un total de 2327 expedientes activos y 412 pasivos, que suman 2803. Se toma como muestra el total de las fuentes, coincidiendo con la población, por lo que se establecen categorías de análisis partiendo de las colecciones documentales organizadas por entidades administrativas de la UCLV, como facultades y centros de investigación. 
Bibliotecas. Vol 35, № 1, enero-junio, 2017, pp.1-20. EISSN: 1659-3286

URL: http://www.revistas.una.ac.cr/index.php/bibliotecas/index

\section{Resultados}

Las tendencias actuales de la conservación abogan por la integración de modelos y métodos que faciliten el diagnóstico atendiendo a las características de la investigación y los tipos de soporte. En la presente investigación se integraron aspectos de dos metodologías encargadas de diagnosticar fondos en archivos y bibliotecas, basadas en los documentos orgánicos tradicionales como el documento de archivo en soporte de papel.

El Modelo de la Biblioteca Nacional de Venezuela tiene una amplia aplicabilidad en instituciones de información. Varios investigadores han validado este método en múltiples investigaciones desarrolladas fundamentalmente en el área latinoamericana y caribeña. El Programas Diagnos del Instituto de Historia de Cuba ha desarrollado diagnósticos de conservación con la capacidad de procesar grandes volúmenes de información. Sobre este método, la investigadora León (2006) afirma que "los diagnósticos pueden realizarse a un grupo de colecciones en conjunto o en una colección determinada de acuerdo a los objetivos que se propongan" (p. 43).

La intervención en los documentos debe realizarse a partir de buenas prácticas investigativas que documenten el estado de conservación de los documentos. Los métodos de diagnóstico de conservación de las colecciones documentales, permiten una lectura aproximada de la realidad. En consonancia a este factor se identifican el estado de conservación y la naturaleza de los daños, mediante la evaluación de sus características y su posterior procesamiento estadístico.

Las organizaciones e instituciones por su actividad generan gran cantidad de documentación. Los archivos de gestión atesoran gran cantidad de fuentes cuya pérdida deviene en perjuicios para la entidad. El diagnóstico de los fondos y las sedes en las que se conservan documentos debe estar acorde con los objetivos y metas de los organismos en los que estos se ejecutan. El diseño de un plan de medidas preventivas puede mitigar los daños que pueda ocasionarse a las fuentes, favoreciendo la mayor perdurabilidad y que se minimicen los riesgos por pérdidas. 


\section{a. Conservación de documentos en el área de FD de la UCLV}

Atendiendo a la variable "sede" se puede establecer que la UCLV se encuentra ubicada en la región central de la República de Cuba. Es un centro de educación superior que forma profesionales en pregrado en más de 50 especialidades de las ciencias técnicas, exactas, sociales y humanísticas (Figura 2). Tiene un amplio programa de formación postgraduada, por lo que genera gran documentación que se almacena en las secretarías de cada facultad y que posteriormente pasa a formar parte de los fondos del archivo central de la universidad.

\section{Figura 2}

\section{Fachada del edificio administrativo de la UCLV donde se encuentra el área de FD}

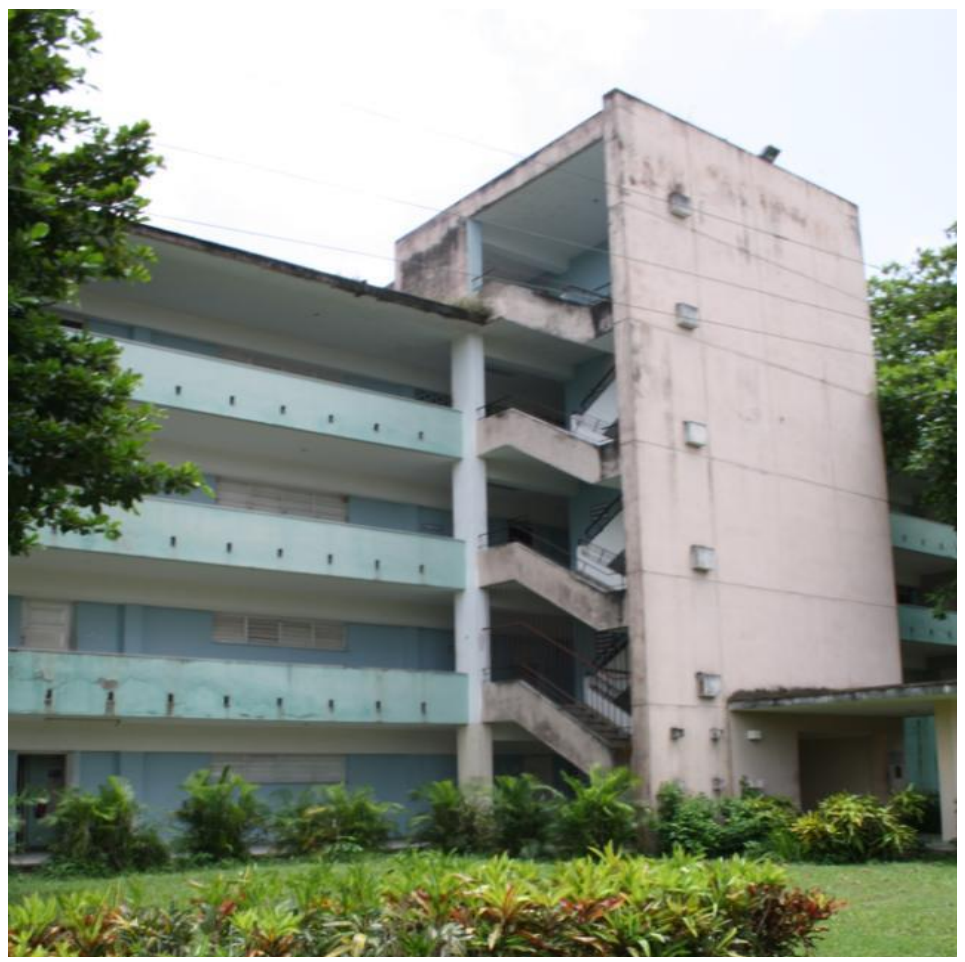

Fuente: Elaboración propia de los autores.

La UCLV fue fundada en 1952, siendo la tercera universidad fundada en Cuba. Las primeras edificaciones de la universidad se levantaron a partir del hormigón armado, el acero laminado y el vidrio plano en grandes dimensiones. Las edificaciones se caracterizan por tener plantas y secciones ortogonales, generalmente asimétricas, ausencia de decoración en las fachadas y grandes ventanales horizontales conformadas por perfiles de 
acero. Las construcciones de la universidad juegan con el relieve ondulado del terreno para definir las diferentes plantas de los edificios. A diferencia de los colegios tradicionales, las edificaciones de la UCLV fueron pensadas para aprovechar la iluminación natural.

La Dirección de Recursos Humanos (DRH) se encuentra ubicada en el segundo piso del Edificio Administrativo conocido como U4 de la Universidad, colinda con la Beca y el Teatro Universitario y al frente tiene presencia de árboles. La DRH tiene como objetivo principal asesorar y orientar a la dirección de la Universidad en establecer, aplicar y controlar una efectiva gestión integrada de recursos humanos en todas las unidades organizativas; así como dirigir y controlar la correcta aplicación de las categorías docentes. En la Figura 3 se puede observar el área de archivo de este departamento y la manera en que está organizada la documentación.

\section{Figura 3}

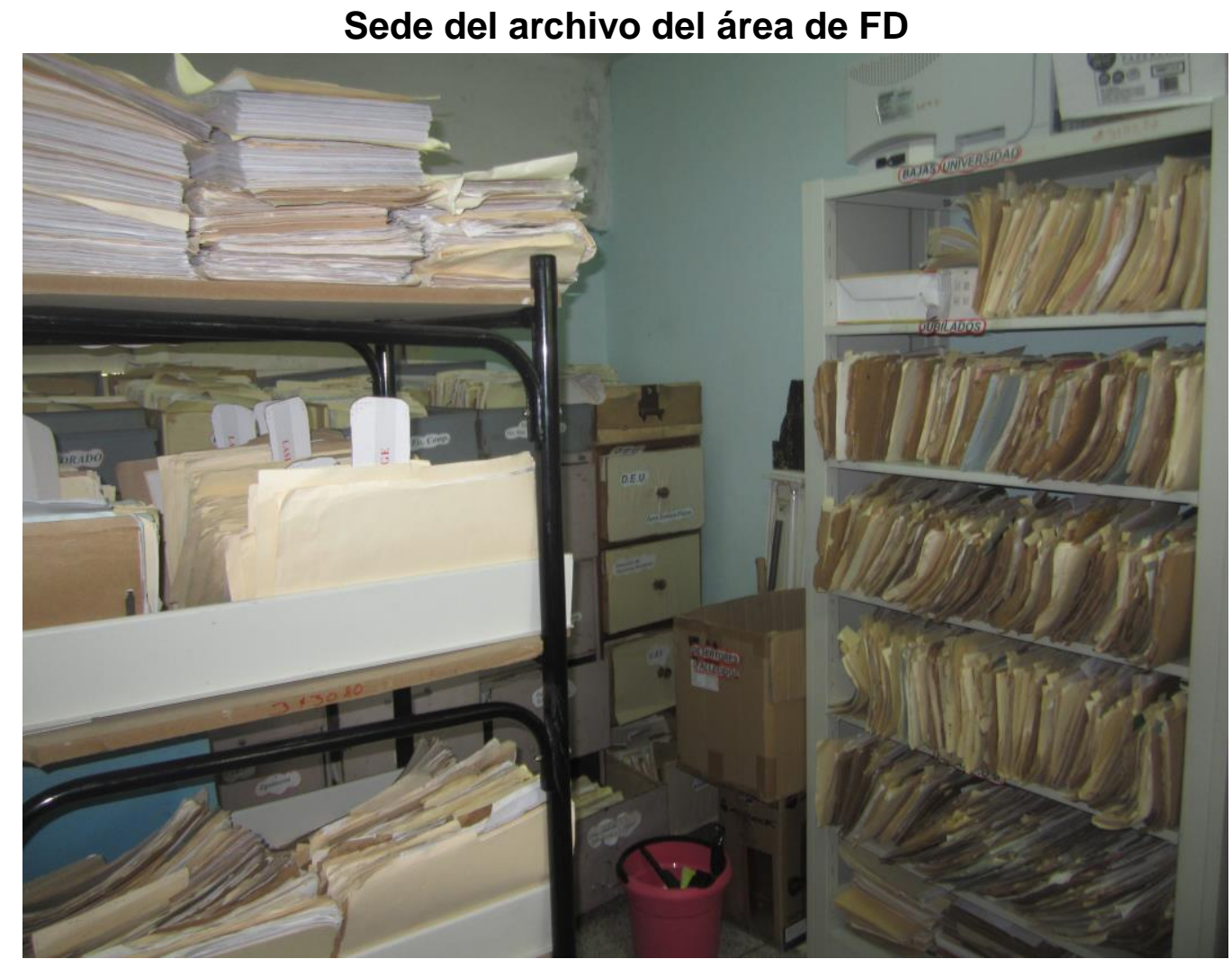

Fuente: Elaboración propia de los autores.

\section{bibliotecas}


El local que ocupa el área de FD se encuentra ubicado al costado derecho del edificio. Esta área se encarga de:

> asesorar la política de los recién graduados en su formación como profesionales,

> elaborar y controlar los planes de capacitación de los trabajadores, los planes de estudio, programas de calificación y recalificación y los programas de selección de la fuerza de trabajo,

> proyectar y desarrollar investigaciones socio-psicológicas,

> estudiar los aspectos sociológicos de la fluctuación de la fuerza de trabajo, las características del proceso de formación y la consolidación de los colectivos laborales,

$>$ proponer a su nivel las cifras en relación con la superación, promoción, evaluación y desarrollo,

> controlar y orientar el proceso de evaluación del desempeño,

$>$ organizar y controlar la ejecución de los planes individuales de desarrollo,

$>$ asesorar y supervisar la correcta aplicación de la estimulación moral a los trabajadores,

> participar en tareas de inspección y control en los temas de planes de trabajo, de capacitación y evaluación del desempeño,

$>$ dirigir y controlar la correcta aplicación del reglamento de categoría docentes y el proceso de los investigadores.

En cuanto a la variable identificación, se establece que el área de FD cuenta con colecciones no bibliográficas relacionadas directamente con la función social que realizan y en correspondencia con la finalidad de un archivo de gestión de dicha envergadura. Se realizó un análisis de la situación actual en cuanto a la conservación de los documentos y se evaluó el estado constructivo del centro, del cual se constatan las condiciones de almacenamiento y se entrevistó a los especialistas del área de FD.

La situación actual que presenta este centro se encauzan en torno a la existencia de una cultura general relacionada con aspectos técnicos formales sobre el desempeño del profesional gestor de los recursos humanos, esto facilita que los procesos de actualización de la documentación sea constante y eficiente; sin embargo, el almacenamiento es deficiente condicionado por aspectos objetivos y subjetivos.

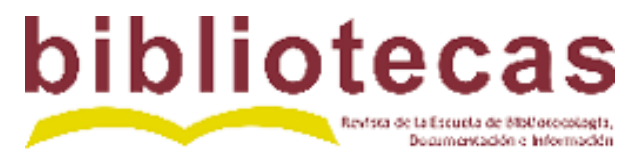


Bibliotecas. Vol 35, № 1, enero-junio, 2017, pp.1-20. EISSN: 1659-3286

URL: http://www.revistas.una.ac.cr/index.php/bibliotecas/index

La ubicación geográfica y el ambiente externo de la institución afectan la conservación de los documentos. A pesar de la preparación de los especialistas existe una carencia de materiales necesarios para garantizar conservación.

La institución se encuentra ubicada dentro del entorno administrativo de la UCLV. La universidad es un centro que tiene una alta presencia de vegetación. Es una zona poco transitada por lo que no existe una baja contaminación por los gases que expulsan los automóviles. La institución está alejada del litoral costero y la misma constituye un punto de reuniones y encuentros de personas que necesitan los servicios que brida el Edificio Administrativo. La institución no está cerca de ríos ni puentes.

Se identifica que las colecciones se encuentran ubicadas en la segunda planta del edificio en un espacio de cinco metros de largo por cuatro de ancho. El depósito se encuentra en un local interior del área de FD que no es compartida para otra función. En el depósito se identifica gran hacinamiento de los documentos. El personal del archivo no cuenta con la formación profesional en cuanto a la organización de archivos y las medidas de conservación que deben manejarse para materiales orgánicos. La institución no cuenta con la estantería suficiente para almacenar los soportes, no poseen equipos controladores del clima y no cuentan con una climatización artificial que posibilite una temperatura estable, constante y que no varíe.

En el Edificio Administrativo frecuentemente ocurren apagones producto del alto voltaje por lo que la temperatura varía con mucha regularidad. Se identifica la carencia de equipos informantes del clima, como termómetros y psicrómetro; de igual forma se carece de deshumificadores, aumentando la humedad relativa ante la presencia de un aire acondicionado. La humedad relativa fluctúa entre el 30 y $70 \%$ en dependencia a la época del año. Se aprecia por las marcas en las paredes la incidencia directa de las lluvias en su lateral.

Los locales no están adecuadamente limpios al igual que la documentación que presenta polvo. La iluminación que se emplea es la artificial y el local está la mayor parte del tiempo 
cerrado. Cuando no funciona la ventilación artificial se debe procurar la mayor ventilación ambiental posible, un local cerrado favorece que microorganismos se reproduzcan y utilicen los documentos como alimento.

Atendiendo a la variable "depósito", el local presenta cuatro ventanas de madera en estado de conservación regular. Se identifica la presencia de una hornilla para cocción de alimentos, elemento que está en contra de las políticas de seguridad y que pudiera causar un incendio en el depósito.

Atendiendo a la variable "mobiliario", se identifica el empleo de 5 estantes metálicos con soporte de cartón de bagazo y con presencia de óxidos ferrosos. El cartón de bagazo es muy degradable y expulsa partículas orgánicas que se alojan en los documentos. Se identifican 13 cajas para el almacenamiento. Las condiciones de almacenamiento no son las correctas, la estantería está cercana a las paredes. Existen altos niveles de hacinamiento propiciado por una estantería insuficiente y el poco espacio disponible. No existen en la sala depósitos para baños o closet. Las condiciones de almacenamiento pude observarse en la Figura 4.

\section{Figura 4}

Almacenamiento de la documentación en el área de FD

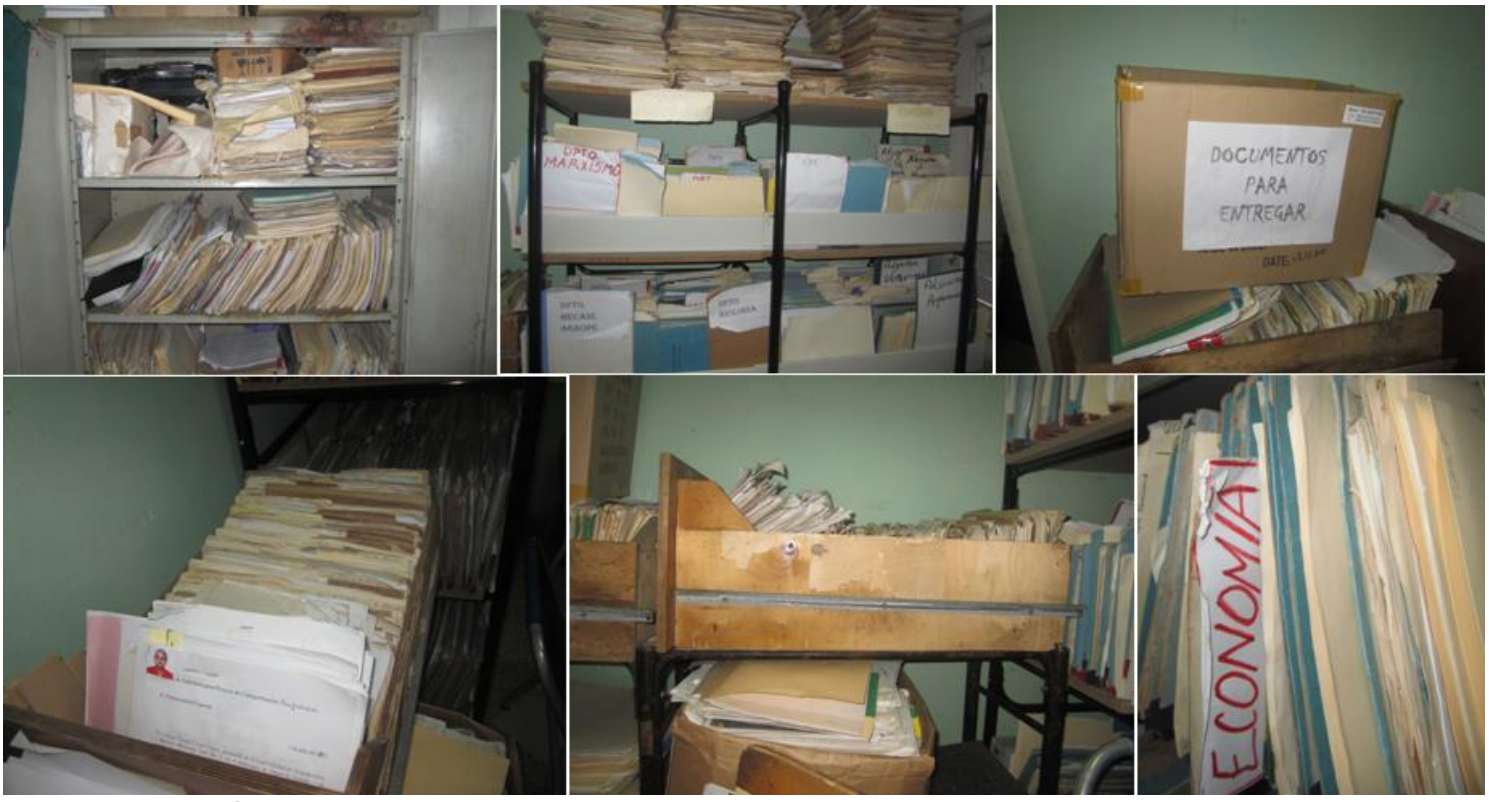

Fuente: elaboración propia de los autores. 
Bibliotecas. Vol 35, № 1, enero-junio, 2017, pp.1-20. EISSN: 1659-3286

URL: http://www.revistas.una.ac.cr/index.php/bibliotecas/index

En cuanto a la variable "condiciones ambientales del depósito", se puede establecer que el depósito está poco ventilado ante la presencia de pocas ventanas. Se emplea la iluminación artificial durante todo el tiempo de trabajo, por lo que el proceso de deterioro aumenta. La afectación de la luz es acumulativa e incide directamente en los documentos. Se emplea un tubo fluorescente en una luminaria sin protector, lo que representa un riesgo, pues las lámparas pueden caerse e iniciar un incendio. En el depósito se almacenan objetos ajenos a la documentación como computadoras e impresoras en desuso, cubos con agua o mobiliario innecesario. En el local se elaboran alimentos por lo que se eleva la humedad relativa y las posibilidades de afectación por insectos y roedores. Existen además recipientes para almacenar agua e implementos para la limpieza como cloro y detergente.

Atendiendo a la variable: condiciones generales de la colección, los documentos carecen de ser procesados según las normas internacionales para el análisis documental. En cuanto a su antigüedad la documentación en sentido general fluctúa entre la década de 1950 hasta la actualidad. Se localizan en el depósito documentos originales que fueron manuscritos en tinta china, pertenecientes a profesores que trabajaron en la universidad desde la década de 1950. Los expedientes que radican en FD poseen actas, declaraciones juradas, autorizaciones de trabajo, certificados de publicación, diplomas, premios, copias de ratificación de categorías docentes y títulos de grados científicos.

Para el diagnóstico de cada documento, se le asignó un número a cada fuente para su ubicación e identificación en la tabla de parámetros realizada. Se efectuó una descripción de los escritos analizados destacando aspectos relacionados con el valor y composición de los documentos. La documentación posee valor probatorio, documental y para algunos expedientes se identifica el valor histórico, y si se elimina puede derivarse prejuicios para la organización, pues se considera información pública.

La tabla de parámetros se realizó a partir de las abreviaturas reflejadas en la Figura 1. El procesamiento estadístico permitió sistematizar varios aspectos de la documentación. El total de documentos está compuesto por pulpa química. Gran parte de la documentación fue elaborada de forma impresa con firmas autorizadas de tipo manuscrito por lo que se 
analiza el tipo de tinta empleado. Se identifica que la tinta más utilizada fue la sintética o comercial.

Se identifica la utilización de tintas ácidas que pueden corroer el papel. Sobre esta afirmación la autora León (2006) corrobora: "las tintas ferrogálicas son preparadas a base de tanino, ácido gálico y sulfato de hierro. Cuando los documentos de soporte papel son sometidos a una humedad relativa alta se descompone por un proceso de hidrólisis y se forman óxidos ferrosos". Se identificaron los principales factores de deterioro de los documentos, los cuales pueden observarse en la Tabla 2, organizados en forma descendente.

Tabla 2

Factores de deterioro identificados en los documentos

\begin{tabular}{|c|c|}
\hline Factores de deterioro & $\begin{array}{c}\text { Cantidad de } \\
\text { documentos }\end{array}$ \\
\hline Polvo & 1053 \\
\hline Amarillamiento & 687 \\
\hline Desprendimiento & 609 \\
\hline Manchas de óxido local & 396 \\
\hline Otras manchas & 387 \\
\hline Emborronado & 306 \\
\hline Manchas de agua & 212 \\
\hline Hongo local & 200 \\
\hline Cinta adhesiva & 142 \\
\hline Acidez & 102 \\
\hline Presencia de insectos & 91 \\
\hline Presencia de excrementos & 31 \\
\hline
\end{tabular}

Fuente: elaboración propia de los autores.

La escritura encima de la cubierta fue realizada por los especialistas que laboran en el área mencionada, quienes emplearon marcadores, lapiceros y plumones. Para el almacenamiento se utiliza una organización regida por la unidad administrativa donde labora el trabajador. En el archivo no se utilizan guías de archivo ni la organización por orden alfabético. La ausencia de formatos de organización aumentan los factores de deterioro físico-mecánicos debido a que el especialista tiene que emplear más tiempo para 
la búsqueda y manipular mayor cantidad de documentos. A pesar de las afectaciones descritas, los documentos son legibles y pueden ser utilizados, por lo que la valoración general de la colección es buena. Algunas de las afectaciones en los documentos pueden observarse en la Figura 5.

\section{Figura 5}

Afectaciones en los documentos

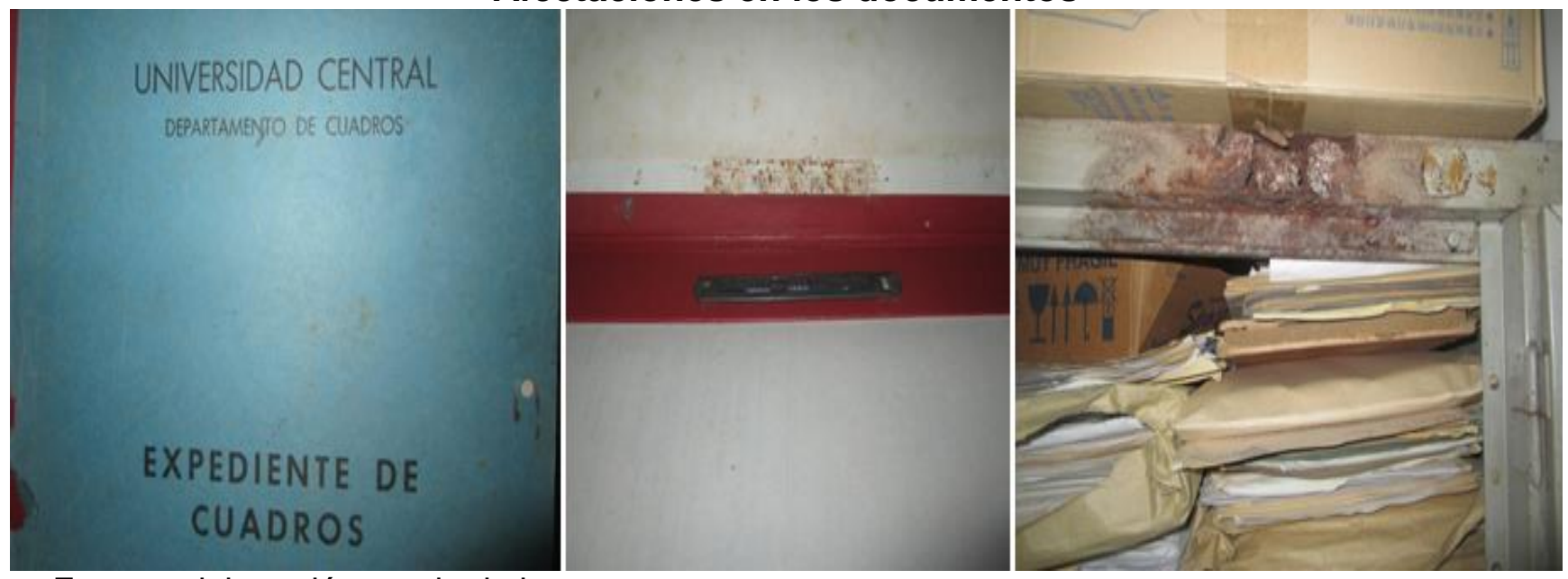

Fuente: elaboración propia de los autores.

La limpieza del local y su entorno no es la adecuada; no se cuenta con medidas de prevención contra catástrofes naturales y antrópicos. Para ejemplificar lo anterior, no hay presencia de extintores, no existe señalización de las áreas con prohibición de fumar, no existe un plan de evacuación de la documentación con valor permanente u otra medida de prevención para garantizar la integridad de la documentación almacenada. Se fumiga contra insectos en varios momentos del año.

Se destaca que a pesar de que la documentación está sometida a procesos continuos de deterioro, por lo general esta posee buen estado de conservación. El factor anterior está determinado a partir de la fecha de la creación de los documentos, pues la mayoría no cuentan con gran antigüedad. Esta situación puede variar en un período de 10 a 15 años, debido a las condiciones físicas y ambientales del depósito. Estos factores combinados con la mala manipulación pueden provocar el deterioro acelerado de la documentación, la cual debe conservarse lo más integralmente posible. La tinta que se emplea para la confección y

\section{bibliotecas}


Bibliotecas. Vol 35, № 1, enero-junio, 2017, pp.1-20. EISSN: 1659-3286

URL: http://www.revistas.una.ac.cr/index.php/bibliotecas/index

actualización de la documentación es sintética o comercial con muy baja calidad por los altos valores de $\mathrm{PH}$ (acidez). Los materiales con que se elaboraron los expedientes laborales son el cartón de escritorio y papel de diferente tipo, entre ellos el papel gaceta de pésima calidad por la acidez que contiene.

\section{Plan de medidas de conservación preventiva para las colecciones documentales que se almacenan en el área de PTP de la DRH de la UCLV}

Luego de haber diagnosticado el estado en que se encuentra el área de FD y las colecciones que almacena, se propone un plan de medidas preventivas con el objetivo de preservar las colecciones y minimizar el deterioro que pueda generarse. Estas medidas se adecuan a las características del local y de la tipologías documentales que en este se encuentran.

1. Acondicionar el local para las funciones que realiza el archivo de gestión de FD. Se deberán eliminar del local todos los objetos ajenos a este y que entorpecen el almacenamiento de la documentación.

2. Ampliar el local o trasladarse a otro de mayor amplitud. El hacinamiento es uno de los factores que actualmente contribuye al mal estado de conservación de las colecciones.

3. Establecer como mínimo dos días al mes para la limpieza de los fondos y tres días a la semana para la limpieza del local.

4. Digitalizar los fondos con alto grado de deterioro y que sean de alto valor para la universidad. De esta forma se evitaría la manipulación innecesaria y por tanto la perdurabilidad de las fuentes originales. Los documentos propuestos para digitalizar deberán ser de profesores que hayan sido reconocidos a nivel nacional e internacional por su labor educativa o científica, además de otros trabajadores que por su actividad (cultural, política, educativa, científica, etc.) resulte relevante, para la historia de la universidad, la conservación de su expediente.

5. Mantener una temperatura estable dentro del local y no exceder los 30 grados centígrados. Se debe garantizar que no se produzcan fluctuaciones de temperatura. Se deberá reparar el sistema eléctrico del edificio o mantener el aire acondicionado permanentemente apagado. 
Bibliotecas. Vol 35, № 1, enero-junio, 2017, pp.1-20. EISSN: 1659-3286

URL: http://www.revistas.una.ac.cr/index.php/bibliotecas/index

6. Adquirir equipos controladores de clima como deshumificador y psicrómetro. Esto debido a que la humedad relativa no debe exceder el $60 \%$ en el local y debe evitarse la ventilación artificial.

7. Establecer un sistema de revisión de fuentes documentales con periodicidad mensual y con muestras diferentes para identificar posibles afectaciones a los documentos por factores biológicos. En caso de infección se recomienda aislar el o los documentos y establecer un período de cuarentena, evitando la contaminación de otros documentos e identificar el tipo de agente biológico para combatirlo.

8. No ingerir o cocinar alimentos en el archivo, los restos de comida atraen a gran variedad de insectos, roedores y microorganismos. Se deberán sacar del local los objetos ajenos al depósito.

9. Evitar ralladuras en los documentos. El especialista deberá velar por la integridad de la fuente documental y exigir el cumplimiento de las normas para el acceso y consulta a la documentación. Las tintas metaloácidas, sintéticas o comerciales son fácilmente degradables, de igual forma la documentación debe conservarse lo más integral posible.

10. Cambiar de mobiliario para almacenar la documentación por una estantería metálica. La estantería de madera es menos duradera y atrae insectos. La estantería metálica a adquirir deberá estar separada $25 \mathrm{~cm}$ de la pared y $50 \mathrm{~cm}$ del suelo, además deberá estar recubierta con un barniz protector con $\mathrm{PH}$ neutro.

11. Limpiar los documentos en seco, utilizando paños de satín para evitar que se adhieran partículas de tejido. De igual forma, se deberán utilizar cepillos de cerdas suaves, siempre en dirección al piso para que el polvo vaya hacia abajo. De ser posible se propone la limpieza con una aspiradora para evitar que los residuos caigan al suelo propiciando el menor movimiento de las partículas.

12. Garantizar la fumigación con los productos idóneos, basado en el material de los fondos y las condiciones ambientales que se presentan en Cuba como país tropical, lo cual constituye un factor determinante para la eliminación de insectos y roedores.

13. Evitar el contacto con otras superficies y el exceso de manipulación. Se deberán realizar las herramientas requeridas para el almacenamiento de la documentación archivística como cuadros de clasificación, plazos de transferencia y guías de archivo.

\section{bibliotecas}


14. Impartir talleres a los trabajadores sobre las características de las fuentes de información que atesoran, la relevancia de la conservación y su influencia para la preservación de la historia y la identidad local y nacional. Además, los talleres deben abarcar la gestión documental y la organización de archivos para garantizar que la documentación se almacene de forma correcta.

15. Realizar un plan contra catástrofes naturales y provocadas, estableciendo una revisión del mismo de forma semestral y solicitando los accesorios necesarios para la protección y evacuación de la documentación.

16. Se propone la confección de una política de acceso a los documentos de la colección, la cual deberá contener los requisitos mínimos para la consulta de fuentes originales en correspondencia con el estado de conservación de la fuente y el objetivo de su consulta.

17. Evitar que la documentación esté cerca de fuentes de calor.

18. Evitar el contacto directo con las fuentes de luz solar. El efecto de la luz es acumulativo y los rayos ultravioletas favorecen la acidez y los cambios químicos del $\mathrm{PH}$ en los documentos compuestos por materiales orgánicos.

19. No poner sustancias adhesivas, no escribir en la superficie de la documentación y no usar pegamentos de cola animal con PH ácido. Esto además del daño a la fuente puede ocasionar una reacción química.

20. Ofrecer y promover el empleo de accesorios que protejan la salud de los usuarios y especialistas que acceden a la colección.

21. Comprobar el estado del documento antes y después de la consulta.

\section{Conclusiones}

Los archivos de gestión almacenan documentos con valor legal y probatorio cuya pérdida puede traer prejuicios a la entidad que los produce. Debe crearse condiciones adecuadas para el almacenamiento de la documentación, fundamentalmente de aquella que pudiera adquirir valor patrimonial en un futuro. Los diagnósticos de conservación documental que combinan varias herramientas garantizan los que los resultados que se obtengan sean lo más certeros posibles. Debe potenciarse la combinación de métodos y procederes para garantizar una adecuada gestión documental enfocada en la conservación preventiva de documentos.

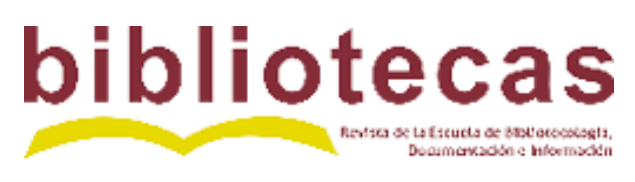


Bibliotecas. Vol 35, № 1, enero-junio, 2017, pp.1-20. EISSN: 1659-3286

URL: http://www.revistas.una.ac.cr/index.php/bibliotecas/index

Los documentos que se atesoran en el área de Formación y Desarrollo de la Dirección de Recursos Humanos que pertenece a la Universidad Central "Marta Abreu" de Las Villas, presenta múltiples afectaciones condicionadas por factores objetivos y subjetivos. Se diseña en plan de medidas preventivas enfocado a la conservación y el almacenamiento de los documentos. El diseño parte de un diagnóstico de conservación documental; garantizando que las medidas se ajusten a las necesidades del centro.

\section{Referencias}

Cabezas, C. (2014). Transparencia activa: Gestión de documentos electrónicos y datos en Chile. En: Serie Bibliotecología y Gestión de Información, 93 (2014): 1-15.

Delgado, A. (2011). Archivar en la nube: reglas de producción del documento contemporáneo. Parte I: Indicadores tecnológicos. En: El profesional de la información, 20 (4): 406-416.

Giménez, V. (2013). UNE-ISO 16175 sobre gestión de documentos en oficinas electrónicas: alcance y limitaciones. En: El profesional de la información, 22(5): 455-458.

Gómez, A.; Dorta, M. y Montes de Oca, L. (2008). Métodos de diagnóstico del estado de conservación de las colecciones documentales Diagnos-Fotodiagnos. Instituto de Historia de Cuba. La Habana: Editora Historia.

González, D.; Térmens, M. y Ribera, M. (2012). Aspectos técnicos de la digitalización de fondos audiovisuales. En: El profesional de la información, 21(5): 520-528.

León, H. (2006). Conservación Preventiva de Documentos. La Habana: Editorial Félix Varela. Muñoz, J. y Nogueras, J. (2014). La digitalización de documentos en la Administración de Justicia. En: Ibersid, 8 (2014): 49-53.

Paz, L.E. y Hernández, E.A. (2015). Conservación de Soportes con Grabaciones Digitales por Medio de la Tecnología Óptica. En: Ciencias Holguín, 2 (2015): 1-14.

Paz, L.E.; Hernández, E.A. y Cuellar, L.L. (2015). Conservación de colecciones documentales de arte. El caso de la Biblioteca Provincial "Marti" de Villa Clara, Cuba. En: Conservamos, 9(9): 8-18.

Paz, L.E.; Hernández, E.A.; Izquierdo, M. y Brito, L. (2016). Fondos documentales del Archivo Parroquial de la Iglesia Católica San Pedro y San Pablo de Corralillo. En: Bibliotecas. Anales de Investigación, 12(1): 41-51. 
Rivera, M.A. (2009). Directrices para la Creación de un Programa de Preservación Digital. En: Serie Bibliotecología y Gestión de Información, 43(2009): 5-61.

Rose, C. (2014). La Conservación Preventiva como Herramienta. España. Disponible en: http://www.ala.archivos.org/-revista/wpcontent/uploads/sites/3/2013/05/Milagros-

Vaillant-Callol-Publicar-190413.pdf (Consultado el 4 de septiembre de 2016).

UNESCO. (2002). Memoria del mundo. Directrices para la salvaguardia del patrimonio documental. París: División de la Sociedad de la Información.

\section{Notas de los autores}

* Luis Ernesto Paz Enrique. Licenciado en Ciencias de la Información. Especialista en Gestión Documental. Dirección de Recursos Humanos. Universidad Central "Marta Abreu" de Las Villas, Cuba. Correo electrónico luisernestope@uclv.cu

** Eduardo Alejandro Hernández Alfonso. Licenciado en Comunicación Social, Profesor del Departamento de Extensión Universitaria, Universidad Central "Marta Abreu" de Las Villas, Cuba. Correo electrónico ealejandro@uclv.cu 